\title{
Orientierende Untersuchungen zur Dauerhaftigkeit von Prosopis kuntzei Harms
}

\author{
G. Scholz • A. Pfeffer • M.S. Ibiza-Palacios • \\ J.V. Oliver-Villanueva
}

Eingegangen: 28. April 2010 / Online publiziert: 26. Februar 2011

(C) The Author(s) 2011. Dieser Artikel ist auf Springerlink.com mit Open Access verfügbar

\begin{abstract}
Zusammenfassung Die Untersuchungen das Kernholzes von Prosopis kuntzei ergaben eine sehr hohe Dauerhaftigkeit gegenüber dem Basidiomyceten Trametes versicolor und der subterranen Termitenart Reticulitermes banyulensis. Im Erdkontakt wird das Holz als mëßig dauerhaft eingestuft. Die niedrigen Masseverluste gegenüber Moderfäule werden neben dem Holzabbau auch Auswaschungsprozessen zugeschrieben.
\end{abstract}

\section{Preliminary study on the durability of Prosopis kuntzei Harms}

\begin{abstract}
The heartwood of Prosopis kuntzei showed excellent resistance against the basidiomycete Trametes versicolor and the subterranean termite species Reticulitermes banyulensis. The wood was classified as moderately durable in soil contact. The low mass losses are presumptively caused by leaching effects in addition to soft rot decay.
\end{abstract}

\section{G. Scholz $(\bowtie) \cdot$ A. Pfeffer}

Holzbiologie und Holzprodukte, Georg-August-Universität

Göttingen, Büsgenweg 4, 37077 Göttingen, Deutschland

e-mail: gscholz@gwdg.de

M.S. Ibiza-Palacios

Department of Wood Technology and Biotechnology, AIDIMA-Wood, Furniture and Packaging Research Institute, Benjamin Franklin 13, 46980 Paterna (Valencia), Spain

J.V. Oliver-Villanueva

Department of Rural Engineering, Polytechnic University of Valencia, Universidad Politécnica de Valencia, Camino de Vera s/n, 46022 Valencia, Spain

\section{Einleitung}

Zugunsten der Schaffung neuer Weideflächen erfolgen großflächige Rodungen im Zentralen Chaco Paraguays (Anonymus 2003). Zur Einbindung des xerophytischen Trockenwaldes in die Landnutzung, beispielsweise als agroforstliches System sowie der Nutzung des Holzes eignen sich verschiedene Baumarten der Gattung Prosopis, u.a. Prosopis kuntzei Harms.

P. kuntzei wurde bereits anatomisch, chemisch und technologisch untersucht (Giménez et al. 1997; Scholz et al. 2005, 2010) und gilt aufgrund seiner Inhaltsstoffe als dauerhaft (Tortorelli 1956; Dimitri et al. 2000; Giménez und Moglia 2003; Scholz et al. 2010). Nach Salvat et al. (2004) weisen in Methanol gelöste Inhaltsstoffe der Rinde antibakterielle Wirksamkeit auf. An wissenschaftlichen Untersuchungen, die Aufschluss über die biologische Dauerhaftigkeit des Kernholzes geben, ist den Autoren nur die Arbeit von Pometti et al. (2010) zum Pilzabbau bekannt. Das Ziel der Arbeit war, das Holz von P. kuntzei gegen Moderfäule, einen holzabbauenden Basidiomyceten, und Termiten zu testen, um über Kennwerte zu verfügen.

\section{Material und Methoden}

\subsection{Holzabbau mit Basidiomyceten}

Die Resistenz gegenüber Trametes versicolor Fr. wurde nach CEN/TS 15083-1 (2005) untersucht. Verwendet wurde Kernholz von P. kuntzei (Loma Plata/Paraguay) und Buche (Dimension: $50 \times 25 \times 15 \mathrm{~mm}^{3}$ und $30 \times 20 \times 10 \mathrm{~mm}^{3}$, Sterilisation: $25 \mathrm{kGy}$ ). Die Berechnung des Masseverlusts (ML) erfolgte nach Formel 1, die der Holzfeuchte (MC) nach Formel $2\left(m_{1}\right.$ : Darrmasse; $m_{2}$ : vom Pilzabbau beeinflusste 
Darrmasse nach Versuchsbeginn; $m_{3}$ : Prüfkörpermasse direkt nach dem Versuchsausbau). Im Rahmen des Artikels ist Formel 2 auf $m_{2}$ und $m_{3}$ bezogen. Die Klassifizierung erfolgte nach CEN/TS 15083-1 (2005) (Klasse 1: sehr dauerhaft; Klasse 5: nicht dauerhaft).

$$
\begin{aligned}
M L & =\frac{m_{1}-m_{2}}{m_{1}} \cdot 100 \% \\
M C & =\frac{m_{3}-m_{1 ; 2}}{m_{1 ; 2}} \cdot 100 \%
\end{aligned}
$$

\subsection{Holzabbau durch Moderfäule}

Die Dauerhaftigkeit im Erdkontakt wurde nach ENV 807 (1999) bestimmt. Je 18 Prüfkörper $\left(100 \times 10 \times 5 \mathrm{~mm}^{3}\right)$ aus Buche und P. kuntzei wurden verwendet. Die Bestimmung des dynamischen Biege-Elastizitätsmoduls $E_{\mathrm{B}} d y n$ (GrindoSonic) erfolgte nach 32 Wochen. Der Feuchtegehalt der Prüfkörper wurde nach Formel 2 bestimmt.

\subsection{Holzabbau durch subterrane Termiten}

Nach EN 117 (2007) wurde im Labor die Resistenz gegenüber der Termitenart Reticulitermes banyulensis CLÉMENT getestet. Drei Kontrollen aus Kiefernsplint wurden zur Prüfung der Virulenz verwendet. Der Test erfolgte in Vermiculit-gefüllten Gläsern (Höhe: $121 \mathrm{~mm}, \varnothing 65 \mathrm{~mm}$ ) bei $70 \pm 5 \%$ relativer Luftfeuchte. Je Glas wurde ein Prüfkörper mit 250 Arbeitern und einer proportionalen Anzahl von Soldaten und Nymphen ausgesetzt, die in Bétera (Valencia, Spanien) gesammelt wurden. Die Auswertung wurde nach EN 350-1 (1994) vorgenommen. Die Einteilung erfolgt dabei in fünf Klassen: 0 (kein Befall) bis 4 (schwerer Befall).

\section{Ergebnisse und Diskussion}

\subsection{Vorbetrachtung}

Gérardin et al. (2004) schreiben die Dauerhaftigkeit von P. africana neben bioziden Extraktstoffen auch hydropho- ben Gumen zu, da die zur Substratbesiedelung notwendige Feuchte fehlt. Das Vorkommen solcher Gumen kann auch für die untersuchte Holzart nicht ausgeschlossen werden (Scholz et al. 2010). In diesem Zusammenhang empfehlen Junga und Militz (2005) u.a. für hydrophobierte Hölzer eine verlängerte Dauer von Pilzabbauversuchen. Im Umkehrschluss wurden, ergänzend zur Norm, neben den normkonformen Prüfkörpern auch die in der Methodik erwähnten, kleineren Testkörper verwendet. Dadurch sollte eine schnellere Substratbesiedlung gewährleistet werden.

\subsection{Holzabbau mit Basidiomyceten}

In Tab. 1 sind die Ergebnisse des Pilzabbauversuchs nach CEN/TS 15083-1 aufgeführt. Die Ausbaufeuchten der Prüfkörper lagen bei durchschnittlich $28 \%$ (P. kuntzei) bzw. $49 \%$ (Buche). In analoger Reihenfolge waren es bei den kleineren Prüfkörperformaten $29 \%$ und $53 \%$. Für T. versicolor gelten nach Weiß et al. (2000) folgende Lebensbedingungen: (5-)24-33(-55) ${ }^{\circ} \mathrm{C}$ bzw. $40 \%$ Holzfeuchte. Die Temperatur liegt aufgrund des Standardklimas mit ca. $22^{\circ} \mathrm{C}$ in einem optimalen Bereich. Die Holzfeuchten der Kontrollen (Buche) bewegen sich zwischen Optimum- und Maximalbereich. Nach CEN/TS 15083-1 ist der Test gültig, da die Masseverluste der Kontrollen für T. versicolor $20 \%$ überschreiten bzw. die Holzfeuchten zwischen $20-80 \%$ betragen.

Nach CEN/TS 15083-1 (2005) liegt der Masseverlust von P. kuntzei unter $5 \%$. Das Kernholz dieser Spezies ist damit sehr dauerhaft (EN 350-1 1994). Diese Resultate stehen im Gegensatz zu Pometti et al. (2010), die für den zentralen Kernholzbereich von P. kuntzei ca. 10,5 \% Masseverlust ermittelten (mäßig dauerhaft). Allerdings handelt es sich vermutlich bei den Masseverlusten von P. kuntzei weniger um abgebaute Holzsubstanz, da ausgewaschene Inhaltsstoffe das die Prüfkörper umgebende Wasser färbten. In diesem Zusammenhang geben Scholz et al. (2010) ca. $15,8 \%$ gelöste Inhaltsstoffe im Kaltwasserextrakt für das Kernholz von P. kuntzei an. Die Masseverluste sind mit anderen Spezies der Gattung Prosopis zu vergleichen. Donoso

Tab. 1 Ermittelte Dauerhaftigkeiten von Prosopis kuntzei gegenüber Weißfäule, Moderfäule und Termiten

\begin{tabular}{|c|c|c|c|c|c|c|c|c|}
\hline \multirow[t]{3}{*}{ Holzart } & \multirow{2}{*}{\multicolumn{2}{|c|}{$\frac{\text { CEN/TS 15083-1 }}{\text { Verlust (\%) }}$}} & \multirow[t]{3}{*}{ Klasse } & \multirow{2}{*}{\multicolumn{2}{|c|}{$\frac{\text { ENV } 807}{\text { Verlust }(\%)}$}} & \multicolumn{3}{|c|}{ EN 117} \\
\hline & & & & & & \multirow[t]{2}{*}{ Befall } & \multirow{2}{*}{$\begin{array}{l}\text { überlebend } \\
(\%)\end{array}$} & \multirow[t]{2}{*}{ Klasse } \\
\hline & Masse Standard & Masse $^{a}$ & & Masse & dyn. E-Modul & & & \\
\hline P. kuntzei & 1,4 & 1,5 & 1 & 6,3 & 23,9 & nein & 0 & 0 \\
\hline F. sylvatica & 27,3 & 47,5 & 4 & 32,8 & 57,1 & - & - & - \\
\hline P. sylvestris & - & - & - & - & - & stark & $80^{\mathrm{b}}$ & 4 \\
\hline
\end{tabular}
Table 1 Determined durabilities of $P$. kuntzei vs. white rot, soft rot and termites

${ }^{a}$ Versuch nach CEN/TS unter Variation der Prüfkörpergröße $\left(30 \times 20 \times 10 \mathrm{~mm}^{3}\right)$

${ }^{b}$ Ein Prüfcontainer wurde aufgrund einer Fremdinfektion nicht berücksichtigt 
et al. (1984) bestimmten folgende Masseverluste für T. versicolor bezogen auf Kernholz: 1,6-2,7 \% (P. tamarugo) bzw. $4 \%$ (P. alba). Carrillo Parra (2007) ermittelte Masseverluste für $P$. laevigata zwischen $0,5-1,2 \%$. Berücksichtigt wurde dabei auch die Auswaschung von 1,68\% an Extrakten.

\subsection{Holzabbau durch Moderfäule}

Die Ergebnisse zum Abbau im Erdkontakt durch Moderfäule sind in Tab. 1 dargestellt. Die Ausbaufeuchten betrugen $34 \%$ (P. kuntzei) und $164 \%$ (Buche). Als Moderfäule bezeichnete Pilze brauchen mehr als $80 \%$ Holzfeuchte zur Substratbesiedelung (Weiß et al. 2000). Diese Feuchten waren bei Buche, nicht aber bei P. kuntzei gegeben. Das kann zum einen an der hydrophoben Wirkung bestimmter Extrakte (Gérardin et al. 2004) sowie an einer geringen Ausgleichsfeuchte des Holzes liegen. Scholz et al. (2010) zeigen, dass phenolische Extraktstoffe nicht nur im Lumen sondern auch in der Zellwand der untersuchten Holzart inkrustiert sind.

Die Masseverluste des Buchenholzes sind nach 32 Wochen vergleichsweise niedrig (z.B. $84 \%$ bei Carrillo Parra 2007), was an den unterschiedlichen Bodensubstraten liegen kann. Dennoch sind die Masseverluste von P. kuntzei fünf mal niedriger als die von nativer Buche.

Im Hinblick auf die Verluste des dynamischen E-Moduls (Tab. 1) werden neben Pilzabbau auch ausgewaschene Inhaltsstoffe vermutet, die nach Harzmann (1988) zur Erhöhung der Holzfestigkeit beitragen. Carrillo Parra (2007) ermittelte für P. laevigata Masseverluste von 10-17 \% bzw. zwischen 18-40\% Verlust des dynamischen E-Moduls nach 32 Wochen. Obwohl P. kuntzei als sehr dauerhaft eingestuft wurde, sind die Masseverluste im Erdkontakt und vor allem der Rückgang des dynamischen E-Moduls höher. Diese Resultate decken sich auch mit den Aussagen für sehr dauerhafte Tropenhölzer wie Teak, die unter den Bedingungen der Gebrauchsklasse 4 und 5 nur mäßig dauerhaft sind (Liese 1979; Schmidt 2006).

\subsection{Holzabbau durch subterrane Termiten}

Die Auswertung des Fraßzwangtests nach EN 117 (2007) ist in Tab. 1 dargestellt. Ein gültiger Test erfordert mehr als $50 \%$ Überlebende bzw. die Einstufung in Klasse 4 nach EN 350-1 (1994) bei den Kontrollen. Diese Bedingungen sind erfüllt, nur Kontrolle 1 wies eine Pilzinfektion im Versuch auf. Dieser Prüfkörper wurde nicht berücksichtigt. Der Test ist damit gültig.

Bei P. kuntzei war ca. eine Woche lang hohe Termitenaktivität zu beobachten. Nach einer weiteren Woche nahm diese Aktivität deutlich ab. Dabei kam es kaum zu einer Beeinträchtigung der Prüfkörper, sodass nach EN 350-1 (1994) das Kernholz als dauerhaft klassifiziert wurde. Dieselbe Klassifizierung liegt auch für $P$. africana vor (Gérardin et al. 2004). Da die Termiten an den Proben aktiv waren, nach Ablauf des Tests jedoch keine Überlebenden mehr vorhanden waren, wird eine biozide Wirkung von Kerninhaltsstoffen vermutet.

\section{Schlussfolgerung}

Die untersuchten Kernholzproben von P. kuntzei zeigen eine hohe biologische Dauerhaftigkeit. Die Holzart lässt Applikationen bis hin zum Erdverbau (Gebrauchsklasse 4) vermuten (z.B. Pfosten, Zäune). Weitere Untersuchungen sind zur Absicherung dieser Erkenntnisse allerdings erforderlich, da nur ein Weißfäulepilz bzw. eine Termitenart verwendet wurden sowie unterschiedliche Provenienzen unberücksichtigt blieben.

Open Access Dieser Artikel unterliegt den Bedingungen der Creative Commons Attribution Noncommercial License. Dadurch sind die nichtkommerzielle Nutzung, Verteilung und Reproduktion erlaubt, sofern der/die Originalautor/en und die Quelle angegeben sind.

\section{Literatur}

Anonymus (2003) Jahrbuch der Geschichte und Kultur der Mennoniten in Paraguay 4:63-64

Carrillo Parra A (2007) Technological investigation of Prosopis laevigata wood from Northeast Mexico. Dissertation, Sierke, GeorgAugust-Universität Göttingen

CEN/TS 15083-1 (2005) Dauerhaftigkeit von Holz und Holzprodukten - Bestimmung der natürlichen Dauerhaftigkeit von Vollholz gegen holzzerstörende Pilze, Prüfverfahren. Teil 1: Basidiomyceten

Dimitri MJ, Leonardis RFJ, Biloni JS (2000) The new book of trees, Bd 1 and II (In Spanish). Manufacturas Editoriales Litográficas. SA $4^{\text {a }}$ Edición, Madrid

Donoso J, Cuevas E, Rosende R, Ulloa I (1984) Natural durability and permeability of Tamarugo (Prosopis tamarugo Phil.) and Algarobo (Prosopis alba Griseb). In: Habit MA (Hrsg) The current state of knowledge on Prosopis tamarugo, Arica, June 11-15 1984, $481 \mathrm{~S}$

EN 117 (2007) Wood preservatives - Determination of toxic values against Reticulitermes species (European termites) (Laboratory method). Beuth, Berlin

EN 350-1 (1994) Durability of wood and wood-based products. Natural durability of solid wood. In: Guide to the principles of testing and classification of natural durability of wood. Part 1. Beuth, Berlin

ENV 807 (1999) Holzschutzmittel. Prüfverfahren für die Bestimmung der Grenze der Wirksamkeit gegen Moderfäule und andere erdbewohnende Mikroorganismen. Beuth, Berlin

Gérardin P, Neya B, Dumarçay S, Pétrissans M, Serraj M, Huber F (2004) Contribution of gums to natural durability of Prosopis africana heartwood. Holzforschung 58:39-44

Giménez AM, Moglia JG (2003) Trees from the Argentinian Chaco. Guide for tree determination. (In Spanish). Facultad de Ciencias Forestales. Universidad Nacional de Santiago del Estero. Secretaría de Ambiente y Desarrollo Sustentable. Ministerio de Desarrollo Social. Editorial El Liberal SRL, Santiago del Estero

Giménez AM, Rios N, Moglia JG (1997) Wood and bark of Prosopis kuntzei (Harms) related to some dendrometric parameters. Agrarias Sistemas y Recursos Forestales 6:163-182 (in Spanish) Investig 
Harzmann LJ (1988) Kurzer Grundriss der allgemeinen Tropenholzkunde. Hirzel, Leipzig

Junga U, Militz H (2005) Besonderheiten bei der biologischen Prüfung einiger modifizierter Holzarten. In: 24. Holzschutztagung der DGFH, Leipzig, S 139-147

Liese W (1979) Die Imprägnierung von Holz für Kühltürme. Holz Roh- Werkst 37:331-335

Pometti CL, Palanti S, Pizzo B, Charpentier J-P, Boizot N, Resio C, Saidman BO (2010) Durability of five native Argentine wood species of the genera Prosopis and Acacia decayed by rot fungi and its relationship with extractive content. Biodegrad. doi:10.1007/ s10532-010-9340-5

Salvat A, Antonacci L, Fortunato RH, Suarez EY, Godoy HM (2004) Antimicrobial activity in methanolic extracts of several plant species from northern Argentina. Phytomedizin 11:230-234
Schmidt O (2006) Wood and tree fungi. Springer, Berlin, S 334

Scholz G, Bues C-T, Bäucker E, Glatzle A (2005) Eigenschaften und Verwendungspotentiale der Baumarten Prosopis kuntzei Harms und Schinopsis cornuta Loes aus dem Chaco Paraguays. Holztechnologie 46:18-25

Scholz G, Windeisen E, Liebner F, Bäucker E, Bues CT (2010) Wood anatomical features and chemical composition of Prosopis kuntzei grown in the Paraguayan Chaco. IAWA J 31:39-52

Tortorelli LA (1956) Timbers and forests from Argentina. Ciencias biológicas y agronómicas. Universidad de Buenos Aires. Ediciones ACME, Buenos Aires (in Spanish)

Weiß B, Wagenführ A, Kruse K (2000) Beschreibung und Bestimmung von Bauholzpilzen. DRW, Leinfelden-Echterdingen, S 126 